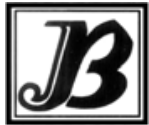

J. bio-sci. 17: 7-12, 2009

ISSN 1023-8654

http://www.banglajol.info/index.php/JBS/index

\title{
DEPOSITION FLUX OF PERFLUORINATED COMPOUNDS IN WET DEPOSITION SAMPLES IN YOKOHAMA, JAPAN
}

\author{
M A Salam¹, M S Hossain ${ }^{2 *}$, M A Haq ${ }^{3}$, M A K Parvez $^{4}$ \\ ${ }_{1}^{1}$ Graduate School of Environment and Information Sciences, Yokohama National University, Japan \\ ${ }^{2}$ Department of Biochemistry and Molecular Biology, Jahangirnagar University, Savar, Bangladesh \\ ${ }^{3}$ Department of Biotechnology and Genetic Engineering, Islamic University, Kushtia, Bangladesh and \\ ${ }^{4}$ Department of Microbiology, Jahangirnagar University, Savar, Dhaka
}

\begin{abstract}
Context: Perfluorinated compounds (PFCs) have emerged as priority environmental contaminants and detected in the environmental and biological matrices concerns due to their persistence and toxicity.

Objectives: To determine the atmospheric deposition of perfluorinated compounds (PFCs) through wet deposition in Japan and measure their environmental levels and deposition flux of wet deposition for assessing the environmental distribution of PFCs.

Materials and Methods: Environmental levels and estimation of deposition flux of perfluorooctane sulfonate (PFOS) and perfluorocarboxylates (PFCAs) were carried out through wet deposition in urban atmosphere of Yokohama, Japan. Solid phase extraction method coupled with high performance liquid chromatography-negative ion electrospray tandem mass spectrometry was used to quantify target PFCs in collected samples.
\end{abstract}

Results: The highest concentration (95.33 ng/L) among target compounds was in snow sample of perfluorooctanoic acid (PFOA). Concentration ranges of perfluorohexanoic acid (PFHxA), perfluoroheptanoic acid (PFHpA), perfluorooctanoic acid (PFOA), perfluorononanoic acid (PFNA), perfluorodecanoic acid (PFDA) and perfluorooctane sulfonate (PFOS) were 0.01-1.86ng/L, 0.01-1.39 $\mathrm{ng} / \mathrm{L}, 0.01-9.02 \mathrm{ng} / \mathrm{L}, 0.07-3.66 \mathrm{ng} / \mathrm{L}, 0.03-0.65 \mathrm{ng} / \mathrm{L}$, and 0-0.47 ng/L, respectively. It was found that 0.6-60 fold differences existed for snow and rain water samples of which PFOA possess highest sixty folds. Statistically significant correlations between various PFCs in wet deposition samples suggested common sources of PFCs in the study area. Wet deposition flux showed monthly variation with increased amount of load during July to September. Total amount of PFCAs in wet fluxes showed composition patterns of PFOA>PFNA>PFHxA>PFHpA>PFDA.

Conclusion: Wet deposition may be a potential transport mechanism of perfluorinated chemicals in the environment.

Key words: Perfluorinated Chemicals, Environmental Level, Deposition flux, Yokohama

\section{Introduction}

Perfluorinated compounds (PFCs) are a class of synthetic compounds used in a variety of industrial applications (Zushi et al. 2010). These compounds are used as polymer additives, lubricants, fire retardants and suppressants, pesticides, and surfactants (Prevedouros et al. 2006, EPA 2007, Zushi and Masuaga 2009). Concern about PFCs, particularly fully fluorinated compounds like perfluorooctane sulfonate (PFOS) and perfluorooctanoic acid (PFOA) have received worldwide attention during last few years. This class of chemicals is also being referred to as "PCBs of the twenty first century" (Taniyasu et al. 2003).

* Corresponding author 
PFCs are resistant to hydrolysis, photolysis, microbial degradation, or metabolism by vertebrates due to high energy carbon-fluorine bond (Giesy and Kannan 2001). PFCs have already been detected in various environmental matrices such as air, water, wildlife, and humans (Saito et al. 2003, Harada et al. 2005. Sinclair and Kannan 2006, Yeung et al. 2006, Nakayama et al. 2007). Many studies suggested the bioaccumulation and toxicity of PFCs (EPA 2003, Martin et al. 2004, Lau et al. 2007). Till today PFOS and PFOA is dominant PFCs in all media. Regulation of these chemicals is time demanding issues in present world. As evidence of the harmful health effects of PFCs piles up, momentum is building in Canada and around the world for a phase-out of these substances. Based on these studies, there is a growing public health concern over the PFCs.

Yokohama is second largest city and heavily industrialized area in Japan. It is situated in Kanto region of Japan where most of the largest industries are located. Until 2000, most of the research on perfluorinated compounds (PFCs) was centered in America and European countries. Japan started research on perfluorinated compounds (PFCs) in 2001. Till today most of the research was carried on water media. Very few researches were conducted on air. The present study is the first approach to determine atmospheric deposition of perfluorinated compounds (PFCs) through wet deposition in Japan. The aim of the present study was to measure environmental levels and deposition flux of wet deposition to provide information for assessing the environmental distribution of PFCs.

\section{Material and Methods}

Standards and reagents: Perfluorohexanoic acid (PFHxA, 97\%) was purchased from Tokyo Kasei Kogyo (Tokyo, Japan). Perfluorooctane sulfonate (PFOS, 98\%), perfluoroheptanoic acid (PFHpA, 96\%), perfluorooctanoic acid (PFOA, 98\%), perfluorononanoic acid (PFNA, 96\%) and perfluorodecanoic acid (PFDA, 96\%) were obtained from Sigma-Aldrich (St. Louis, MO, USA). Ultrapure water (Millipore Milli-Q water) was used throughout the experiments. Methanol, accetonitrile and acetone (HPLC grade) and ammonium acetate were purchased from Wako Chemicals Industries Ltd (Osaka, Japan). The concentrations of the target PFCs were not found in the methanol used concentrated by the same amount as unknown samples.

Collection of wet deposition sample: The study was carried out at Yokohama National University. It was situated at $35^{\circ} 28^{\prime \prime} \mathrm{N}$ and $139^{\circ} 35^{\prime \prime} \mathrm{E}$. The study area was considered as an urban area of Japan. The sampling location is shown in Fig. 1. Wet deposition implies rain water and snow. Monthly rain water sampling was carried out from January to December, 2006 except April and May. Automatic rain water sampler was used to collect the sample and was set at the roof top of four storied building of Environment and Information Sciences Building No.4 at Yokohama National University. Methanol rinsed stainless steel pot with an inner diameter of $30 \mathrm{~cm}$ were used for collection of rain water. The pots of the sampler were placed above $120 \mathrm{~cm}$ of rooftop to prevent entering foreign matters. Polypropylene containers with narrow mouth bottles screw tops were used to store samples in refrigerator at $-4^{\circ} \mathrm{C}$ temperature until analysis. Prior to storage containers were rinsed with methanol and ultrapure water (Millipore Milli-Q water). Teflon bottles and Teflon lined caps were avoided throughout the experiment to prevent sample contamination. Snow sample was collected during snowfall by using pre-rinsed stainless steel pot in the same rooftop and was brought to laboratory immediately after collection. Samples were packed in polyethylene container and transported to the laboratory. Ultrapure water was stored in the same type of container to assess potential contamination. Tsurumi river basin is the main water outlet which is carries out discharged water into the Tokyo Bay.

To compare the concentration levels in rain water, river water and snowfall, data of Tsurumi river measured by Takeda et al. (2006) was considered. 


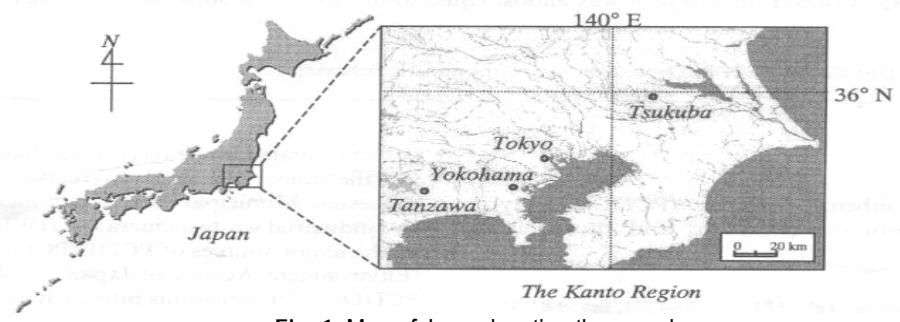

Fig. 1. Map of Japan locating the sample area

Extraction: Rain water and snow samples together called wet deposition were extracted by using a dual piston positive pressure pump system (Waters Sep-Pak Concentrator Plus, Waters Corporation). Preconditioned Oasis HLB $500 \mathrm{mg} / 6 \mathrm{cc}$ cartridges (Waters Corporation, Milford, MA) was equipped with the glassfiber filter paper (the size: $\varphi 47 \mathrm{~mm}$ and hole diameter $1 \mu \mathrm{m}$ ) to remove the suspended materials or insoluble particles. Before loading the samples, the cartridges were preconditioned with $10 \mathrm{ml}$ of methanol and ultrapure water (Millipore Milli-Q water). The sample container was inverted to mix thoroughly and measure $1 \mathrm{~L}$ of sample using glass measuring flux. Samples were then passed through Oasis HLB $500 \mathrm{mg} / 6 \mathrm{cc}$ cartridges column at a flow rate of $10 \mathrm{ml} / \mathrm{min}$. The cartridges were then centrifuged for 10 minutes at $1000 \mathrm{rpm}$, eluted with $7 \mathrm{~mL}$ methanol and evaporated at $40^{\circ} \mathrm{C}$ temperature under gentle nitrogen purge. Again $1 \mathrm{ml}$ of methanol was added to get analyte for analysis and it was filtration using nylon syringe filter (The pore size $0.2 \mu \mathrm{m}$ and made of millipore) to make clear elute.

Analysis and quantification: Separations of analytes were performed by Agilent HP1100 liquid chromatogram (Agilent Palo Alto, CA) interfaced with a Micromass Quattro-Ultima spectrometer (Waters Corp. Milford, MA) operated in electrospray negative ionization mode. A $10 \mu \mathrm{L}$ aliquot of the sample extract was injected into a Zorbax XBD C-18 (Agilent Narrow Bore $2.1 \mathrm{~mm}$ i.d. x $150 \mathrm{~mm}, 5 \mu \mathrm{m}$ ) column at a flow rate of $0.2 \mathrm{~mL} / \mathrm{min}$ with $10 \mathrm{mM}$ ammonium acetate aqueous solution (solvent A) and acetonitrile (solvent B) at $55: 45$ to obtain specified retention time. The total run time was 30 min, without any equilibration time between samples. Mass spectra were obtained using an Agilent 1100 orthogonal ion spray MSD, employing a negative electrospray ionization mode. The nebulizer pressure was $50 \mathrm{psi}$ and the drying nitrogen gas flow was set at $10 \mathrm{l} / \mathrm{min}$. The selected ion monitoring (SIM) mode was used for quantification of analyte and the monitoring ions for each analyte were listed in Table 1.

Quality assurance/quality control: Contamination during sampling, extraction, and analysis was examined by analyzing blank samples of ultrapure water treated in the same manner as the real samples. Blank sample was analyzed every 10 real samples. The blank samples were not detected quantifiable PFC. Recoveries of the analytes in wet deposition samples were determined by addition of $100 \mathrm{ng}$ standard to $1-\mathrm{L}$ ultrapure water. Five replicates for PFCs spike concentrations were analyzed. The recoveries for all analytes were in the range of $65-110 \%$. A calibration was performed for each set of experiment using dilutions of an external standard stock solution of PFCs prior to real samples analysis.

Table 1. Name, acronym, structure and monitoring of targets PFCs compounds

\begin{tabular}{lccc}
\hline Name of Compound & Acronym & Formula & Monitoring ion $(\mathrm{m} / \mathrm{z})$ \\
\hline Perfluoroctane Sulsonate & PFOS & $\mathrm{CF}_{3}\left(\mathrm{CF}_{2}\right)_{7} \mathrm{SO}_{3-}{ }^{-}$ & 499,500 \\
Perfluorohexanoic acid & PFHxA & $\mathrm{CF}_{3}\left(\mathrm{CF}_{2}\right)_{4} \mathrm{CO}_{2}{ }^{-}$ & 313,269 \\
Perfluoroheptanoic acid & PFHpA & $\mathrm{CF}_{3}\left(\mathrm{CF}_{2}\right)_{5} \mathrm{CO}_{2}^{-}$ & 363,319 \\
Perfluorooctanoic acid & PFOA & $\mathrm{CF}_{3}\left(\mathrm{CF}_{2}\right)_{6} \mathrm{CO}_{2-}^{-}$ & 413,369 \\
Perfluorononanoic acid & PFNA & $\mathrm{CF}_{3}\left(\mathrm{CF}_{2}\right)_{7} \mathrm{CO}_{2-}^{-}$ & 463,419 \\
Perfluorodecanoic acid & PFDA & $\mathrm{CF}_{3}\left(\mathrm{CF}_{2}\right)_{8} \mathrm{CO}_{2-}^{-}$ & 513,469 \\
\hline
\end{tabular}




\section{Results and Discussion}

Environmental levels of PFCs in wet deposition: Concentration of target perfluorocarboxylates (PFCAs) and perfluorooctane sulfonate (PFOS) in wet deposition were summarized in Table 2. Among the target compounds concentration ranges were varied from $0.01-95.33 \mathrm{ng} / \mathrm{l}$. In snow sample, higher concentrations were $95.33 \mathrm{ng} / \mathrm{l}$ and $2.90 \mathrm{ng} / \mathrm{l}$ in PFOA and PFDA, respectively. Zushi et al. (2008) recently reported that total PFCs load of river is significant. For rain water, over the whole analysis, concentration ranges of perfluorohexanoic acid (PFHXA), perfluoroheptanoic acid (PFHpA), perfluorooctanoic acid (PFOA), perfluorononanoic acid (PFNA), perfluorodecanoic acid (PFDA) and perfluorooctane sulfonate (PFOS) were 0.01-1.86ng/l, 0.01-1.39 ng/l, 0.01-9.02 ng/l, 0.07-3.66 ng/l, 0.03-0.65 ng/l, and 0.01-0.47 ng/l, respectively. Highest concentration of PFHxA, PFHpA, PFOA, PFNA, PFDA and PFOS was found in rain water in the months of September, August, January, September, August and September, respectively. The presence of PFCAs and PFOS in rainfall and snow indicates the atmospheric presence of PFCAs and PFOS in wet deposition samples in the study area. This could be the result of the atmospheric transportation of PFCAs themselves or volatile precursor's fluorutelomer alcohol which undergo transformation to PFCAs and PFOS or the result of point sources nearby study area. Concentration between snow and rain water samples were found more than 0.57-60 fold differences and it was highest in PFOA sixty folds.

Concentrations of rain water and event snow fall for present study and nearest river of the study area namely Tsurumi River, water concentrations (Takeda 2006) were used for comparison of the results. These comparisons were summarized in Table 3. Except PFOA, river water contains higher concentrations than rain water and snow. PFOA concentration in snow was $95.33 \mathrm{ng} / \mathrm{l}$. Concentrations of PFCs in river water was relatively higher than rainwater and snow. This is because river water has direct (waste water treatment plant, industry along the bank of rivers etc.) and indirect sources (human activities in river) which are contributing PFCs continuously. Highest concentration of river water was found in PFOS (202 ng/l) but it was lowest in rain water $(0.12 \mathrm{ng} / \mathrm{l})$. This difference indicates that solubility of PFOS varies based on water nature. US EPA reported the solubility of PFOS in pure water and unfiltered seawater was $570 \mathrm{mg} / \mathrm{l}$ and 12.4 $\mathrm{mg} / \mathrm{l}$, respectively (EPA 2003). Compositional trend of these three media were completely different from each other. It was PFOS $>$ PFNA $>$ PFOA $>$ PFHXA $>$ PFDA $>$ PFHpA, PFOA $>$ PFNA $>$ PFHXA $>$ PFHpA $>$ PFDA $>$ PFOS and PFOA $>$ PFDA $>$ PFNA $>$ PFHXA $>$ PFHpA $>$ PFOS for Tsurumi river water, rain water and snow sample, respectively.

Correlation among PFC in rainwater: Correlations between different PFCs have been used to explain the sources of PFCs. Concentrations of PFCs in rainwater samples were used for correlation analysis of the present study. Among PFCAs, statistically significant correlations were found between PFHxA, PFOA, PFNA and PFDA. The strongest correlation was found between PFDA and PFOA $(r=0.97 ; N=10)$ in rainwater samples. These findings support the hypothesis that telomer alcohols could degrade to yield various perfluorinated acids of PFHXA, PFOA, PFNA and PFDA and other short chain carboxylic acid in the study area. FTOHs break down in the atmosphere to give the fluorotelomer acids of 10:2: 8:2; and 6:2 and these fluorotelomer acids undergo degrade to form the longer chain length of PFCAs (Ellis et al. 2003). Similarly, PFOS was found to be positively correlated between PFOS:PFHXA ( $r=0.50 ; N=10)$ and PFOS:PFHpA ( $r=$ $0.77 ; N=10)$ and Significant correlation was found between PFOS and PFHpA $(r=0.77 ; N=10)$. These results suggest that there might be common sources of PFHXA, PFHpA, and PFOS in the study area. Good agreement was found with the previous study (Yeung et al. 2006) in which a positive significant correlation was found between PFOS and PFHxS concentrations in human blood samples.

Deposition flux of PFCs in wet deposition: The deposition flux of PFCs in urban Yokohama, Japan was calculated daily basis. It was calculated by using the following formula: $D F=C \times Q /(A) /(D)$. Here, DF= Deposition flux, $C=$ concentration, $Q=$ Amount of rain/amount of dry deposition, $(A)=$ Area of sample bucket 
and $(D)=$ Duration of sample period. Deposition flux of perfluorocarboxylic acids (PFCAs) and perfluorooctane sulfonate (PFOS) were presented in Table 4. Total and average daily depositional fluxes were estimated to be $36 \mathrm{ng} / \mathrm{m}^{2} / \mathrm{d}, 22 \mathrm{ng} / \mathrm{m}^{2} / \mathrm{d}, 345 \mathrm{ng} / \mathrm{m}^{2} / \mathrm{d}, 48 \mathrm{ng} / \mathrm{m}^{2} / \mathrm{d}, 23 \mathrm{ng} / \mathrm{m}^{2} / \mathrm{d}, 7 \mathrm{ng} / \mathrm{m}^{2} / \mathrm{d}$ and $4 \mathrm{ng} / \mathrm{m}^{2} / \mathrm{d}$, $2 \mathrm{ng} / \mathrm{m}^{2} / \mathrm{d}, 34 \mathrm{ng} / \mathrm{m}^{2} / \mathrm{d}, 5 \mathrm{ng} / \mathrm{m}^{2} / \mathrm{d}, 2 \mathrm{ng} / \mathrm{m}^{2} / \mathrm{d}$ and $0.7 \mathrm{ng} / \mathrm{m}^{2} / \mathrm{d}$ for PFHXA, PFHpA, PFOA, PFNA, PFDA and PFOS, respectively. Large variation among targets compounds were exhibited having more than 50 folds in month profiles. Deposition fluxes of rain water intended to show increased amount during the months of July to September and December. This sort of variation on the deposition fluxes could be ascribed to different absorption capacities of target compounds. In total depositional fluxes, the compositional pattern of PFCAs was PFOA>PFNA>PFHXA>PFDA>PFHpA. For PFOA, the highest depositional flux was $\left(266 \mathrm{ng} / \mathrm{m}^{2} / \mathrm{d}\right)$ found in snow samples. Present study is consistent with the result of Saito et al. (2003). They showed PFOS in dust particle to have higher concentration during July to August. This is the first approach to determine deposition flux in rain water and snow. So data for global scale could not be compared.

Table 2. Environmental levels (ng/l) of PFCs in rainwater and snow samples in Yokohama

\begin{tabular}{lcccccc}
\hline Month & PFHxA & PFHpA & PFOA & PFNA & PFDA & PFOS \\
\hline January & 0.01 & 0.02 & 9.02 & 0.50 & 0.41 & 0.09 \\
February* & 0.38 & 0.22 & 95.33 & 1.27 & 2.90 & 0.16 \\
March & 0.31 & 0.16 & 0.23 & 0.07 & 0.03 & 0.01 \\
June & 0.04 & 0.01 & 0.04 & 0.18 & 0.11 & 0.01 \\
July & 0.96 & 0.77 & 0.00 & 0.13 & 0.03 & 0.02 \\
August & 0.94 & 1.39 & 1.60 & 0.68 & 0.65 & 0.38 \\
September & 1.86 & 0.91 & 2.04 & 1.69 & 0.41 & 0.47 \\
October & 1.26 & 0.01 & 0.02 & 1.31 & 0.36 & 0.02 \\
November & 1.41 & 0.01 & 0.03 & 3.66 & 0.47 & 0.04 \\
December & 0.08 & 0.11 & 1.04 & 0.34 & 0.07 & 0.08 \\
\hline * February repr
\end{tabular}

${ }^{*}$ February represent snow sample

Table 3. Comparison of PFCs (ng/l) in river water, rain water and snow

\begin{tabular}{lcccccc}
\hline Source & PFHXA & PFHpA & PFOA & PFNA & PFDA & PFOS \\
\hline${ }^{*}$ Riverwater & 6.90 & 3.60 & 16.00 & 8.30 & 3.70 & 202.20 \\
Rain water & 0.76 & 0.38 & 1.55 & 0.95 & 0.28 & 0.12 \\
Snow & 0.38 & 0.22 & 95.33 & 1.27 & 2.90 & 0.16 \\
\hline *River water-Takda & 2006 & & & &
\end{tabular}

*River water-Takeda, 2006

Table 4. Deposition flux $\left(\mathrm{ng} / \mathrm{m}^{2} / \mathrm{d}\right)$ of wet deposition in Yokohama

\begin{tabular}{lcccccc}
\hline Month & PFHxA & PFHpA & PFOA & PFNA & PFDA & PFOS \\
\hline January & 0.08 & 0.13 & 53.68 & 2.99 & 2.43 & 0.54 \\
February & 1.05 & 0.60 & 265.94 & 3.55 & 8.03 & 0.44 \\
March & 1.40 & 0.72 & 1.01 & 0.33 & 0.13 & 0.00 \\
June & 0.37 & 0.00 & 0.38 & 1.83 & 1.09 & 0.00 \\
July & 6.07 & 4.86 & 0.00 & 0.81 & 0.20 & 0.11 \\
August & 7.90 & 11.62 & 13.18 & 5.66 & 5.41 & 3.15 \\
September & 7.59 & 3.74 & 8.34 & 6.93 & 1.66 & 1.94 \\
October & 2.27 & 0.02 & 0.03 & 2.37 & 0.65 & 0.03 \\
November & 8.92 & 0.07 & 0.18 & 23.16 & 2.96 & 0.27 \\
December & 0.15 & 0.20 & 1.98 & 0.64 & 0.14 & 0.15 \\
Average & 3.58 & 2.20 & 34.47 & 4.83 & 2.27 & 0.66 \\
\hline Total & 35.81 & 21.97 & 344.73 & 48.29 & 22.70 & 6.63 \\
\hline
\end{tabular}




\section{Conclusions}

Present study focused on the assessment of environmental levels of PFHxA, PFHpA, PFOA, PFNA, PFDA and PFOS in wet deposition from the urban atmosphere of Yokohama, Japan. Environmental levels of PFCs were detected in all the samples designed so far. Snow contained highest concentration of PFOA compared to rainwater samples. Strong positive correlations among PFCAs and PFOS suggested that target compounds could have been derived from common sources in the study area. It shows the evidence that rainwater and snowfall may be a potential transport mechanism of PFCs in the environment. It is also suggested that FTOHs which are used as aid material in different industrial and commercial purpose undergo degradation in atmosphere to yield different short and long chain PFCAs and transported in the study area.

\section{References}

Ellis DA, Martin JW and Mabury SA, Hurley MD, Sulbaek MP, Wallington TJ. 2003. Atmospheric lifetime of fluorotelomer alcohols. Environ Sci Technol 37, 3816-3820. doi:10.1021/es034136j

Giesy JP, Kannan K. 2001. Global distribution of perfluorooctane sulfonate in wildlife. Environ Sci Technol 35, 1339-1342

Harada K, Nakanishi S, Saito N, Tsutsui T, Koizumi A. 2005. Airborne perfluorooctanoate may be a substantial source contamination in Kyoto area, Japan. Bull Environ Contam Toxicol 74, 64-69. doi:10.1007/s00128-004-0548-0

Lau C, Anitole K, Hodes C, Lai D, Pfahles-Hutchens A, Seed J. 2007. Perfluoroalkyl acids: a review of monitoring and toxicological findings. Toxicol Sci 99, 366-394. doi:10.1093/toxsci/kfm128

Martin JW, Whittle DM, Muir DC, Mabury SA. 2004. Perfluoroalkyl contaminants in a food web from Lake Ontario. Environ Sci Technol 38, 5379-5385. doi:10.1021/es049331s

Nakayama S, Strynar MJ, Helfant L, Egeghy P, Ye X, Lindstrom AB. 2007. Perfluorinated compounds in the Cape Fear drainage basin in North Carolina. Environ Sci Technol 41, 5271-5276. doi:10.1021/es070792y

Prevedouros K, Cousins IT, Buck RC, Korzeniowski SH. 2006. Sources, fate and transport of perfluorocarboxylates. Environ Sci Technol 40, 32-44. doi:10.1021/es0512475

Saito N, Sasaki K, Nakatome K, Harada K, Yoshinaga T, Koizumi A. 2003. Perfluorooctane sulfonate concentrations in surface water in Japan. Arch Environ Contam Toxicol 45, 149-158. doi:10.1007/s00244-003-0163-9

Scott BF, Spencer C, Mabury SA, Muir DCG. 2006. Poly and perfluorinated carboxylates in North American precipitation. Environ Sci Technol 40, 7167-7174. doi:10.1021/es061403n

Sinclair E, Kannan K. 2006. Mass loading and fate of perfluoroalkyl surfactants in wastewater treatment plants. Environ Sci Technol 40, 1408-1414. doi:10.1021/es051798v

Takeda T. 2006. Mass balance of perfluorofurfactants in Tokyo Bay, Japan. Master thesis. Submitted to the Graduate School of Environment and Information Sciences, Yokohama, Japan, $90 \mathrm{pp}$.

Taniyasu S, Kannan K, Horii Y, Hanari N, Yamashita N. 2003. A survey of perfluorooctanesulfonate and related perfluorinated organic compounds in water, fish, birds and humans from Japan. Environ Sci Technol 37, 2634-2639.

U.S. Environmental Protection Agency. 2003. Preliminary risk assessment of the developmental toxicity associated with exposure to perfluorooctanoic acid and its salts, U.S. Environmental Protection Agency, Washington, DC.

U.S. Environmental Protection Agency. 2007. Perfluoroalkyl sulfonates; significant new use rule. In: US Environmental Protection Agency, (ed.) US Environmental Protection Agency: Washington, DC, 72, 57222-57235 pp.

Yeung LW, So MK, Jiang G, Taniyasu S, Yamashita N, Song M. 2006. Perfluorooctanesulfonate and related fluorochemicals in human blood samples from China. Environ Sci Technol 40, 715-720. doi:10.1021/es052067y

Zushi Y, Masunaga S. 2009. First-flush loads of perfluorinated compounds in stormwater runoff from Hayabuchi River basin, Japan served by separated sewerage system. Chemosphere 76(6), 833-40.

Zushi Y, Takeda T, Masunaga S. 2008. Existence of nonpoint source of perfluorinated compounds and their loads in the Tsurumi River basin, Japan. Chemosphere 71(8), 1566-73. doi:10.1016/j.chemosphere.2007.11.055

Zushi Y, Tamada M, Kanai Y, Masunaga S. 2010. Time trends of perfluorinated compounds from the sediment core of Tokyo Bay, Japan (1950s-2004). Environ Pollut 158(3), 756-63. doi:10.1016/j.envpol.2009.10.012 